\title{
Metastatic Liver Tumors in Surgical Pathology: Impact of Contemporary Diagnostic and Therapeutic Paradigms in a Tertiary Care Center
}

International Journal of Surgical Pathology I-7

(C) The Author(s) 2021

Article reuse guidelines:

sagepub.com/journals-permissions DOI: $10.1 \mid 77 / 10668969211022708$

journals.sagepub.com/home/ijs

(S)AGE

\author{
Ayşe Armutlu, MD' (D, Omer Saeed, MD², \\ and Romil Saxena, MBBS, MD, FRCPath ${ }^{2}$
}

\begin{abstract}
We analyzed metastatic liver tumors received in the department of pathology in a tertiary care center over a 3-year period. There were 509 metastatic liver tumors; counterintuitively, there were as many resections ( 235 cases) as biopsies ( 274 cases). This unexpected finding reflects contemporaneous organ-specific paradigms for diagnosis and management of metastatic liver disease in oncologic practice, and the association of our practice with a National Cancer Institute-designated comprehensive cancer center with expertise and specialization in liver surgery. We receive a large number of resections for metastatic liver tumors because metastasectomy from a variety of primary tumors is associated with improved overall, and in many instances, disease-free, long-term survival. Metastatic colorectal carcinomas, metastatic neuroendocrine tumors, and metastatic gastrointestinal stromal tumors constituted $78 \%$ of resections because the largest body of literature and cumulative experience exists for these lesions. In contrast, breast carcinomas and pancreatic carcinomas, which are the next common metastatic liver tumors were biopsied but rarely resected, because metastasectomy is not the standard of care for these tumors. Immunohistochemistry was performed in less than a quarter of the total number of cases $(23 \%)$, because the primary tumor site was known in the vast majority of cases. Of the 42 cases with unknown primary tumor, it was elucidated in $50 \%$ of the cases by immunohistochemical and clinical work-up. Of the cases with known primary tumor, immunohistochemistry was performed mostly in metastatic breast, colon, and lung carcinomas. In these cases, biomarker analyses provided additional information relevant to clinical management.
\end{abstract}

\section{Keywords}

metastatic liver tumors, metastatic neuroendocrine carcinoma, metastatic colorectal carcinoma, metastatic gastrointestinal stromal tumor, liver transplantation, immunohistochemistry

\section{Introduction}

The liver is a common site for metastases from tumors originating in various organs of the body, and metastatic liver tumors are a common specimen type in surgical pathology. There are several studies of the incidence and pattern of liver metastases from a particular organ or organ system, based on records in Surveillance, Epidemiology and End Results (SEER) database. There are also several studies that address the challenges of microscopic diagnosis of metastatic liver tumors, and particularly the utility of immunohistochemistry (IHC) in the differential diagnosis. However, there is scant literature on the incidence and clinico-pathologic characteristics of metastatic liver tumors seen on the whole in surgical pathology practice. We analyzed consecutive cases of metastatic liver tumors received in the department of pathology over a 3 years period from our institution, which is a tertiary care center with a National Cancer Institute (NCI)-designated comprehensive cancer center, with additional specialization in liver surgery.

\section{Materials and Methods}

Following approval by the Institutional Review Board of Indiana University, the pathology database was searched for all metastatic liver tumors in adult patients received from our institution over a 3-year period. Primary and

\footnotetext{
'Koç University, Istanbul, Turkey

${ }^{2}$ Indiana University School of Medicine, Indianapolis, IN, USA

Corresponding Author:

Romil Saxena, Indiana University Health Pathology Laboratory, 350 West II th street, Room 40I4, Indianapolis, IN 46202, USA.

Email: rsaxena@iupui.edu
} 
benign liver tumors, pediatric patients, and consultation cases from outside institutions were excluded. Twenty-seven needle biopsies diagnosed as metastatic adenocarcinoma were excluded after review of the medical charts indicated that there was no extra-hepatic primary tumor, and that the final clinical diagnosis was deemed to be intrahepatic cholangiocarcinoma.

Following the identification of the patients, data was extracted from pathology reports and medical records. The data points recorded were age, gender, site of the primary tumor, multifocality, type of resection, resection margins, pathologic diagnosis, IHC stains, and molecular testing performed. We also recorded whether at the time of liver biopsy or resection, the site of the primary tumor was known clinically, or by prior biopsy, or whether the primary tumor was resected concurrently. In cases where the primary site was not known at biopsy or resection, the records were reviewed to determine how the primary tumor site was eventually diagnosed.

\section{Results}

There were 509 metastatic liver tumors over the 3-year study period compared to 260 primary malignant liver tumors (188 hepatocellular carcinomas, 68 cholangiocarcinomas, and 4 combined hepatocellular-cholangiocarcinomas). Patients with metastatic liver tumors ranged in age from 18 to 89 years (average, 59; median, 61 years). There were 260 (51\%) females and $249(49 \%)$ males. The 5 commonest metastatic liver tumors (Table 1) were colon adenocarcinomas (192 cases, 38\%), neuroendocrine tumors (54 cases, $10.6 \%$ ), breast carcinomas (50 cases, $9.8 \%$ ), pancreatobiliary carcinomas (48 cases, 9.4\%), and tumors of the female genital tract (27 cases, 5.3\%). These were followed by lung carcinomas (18 cases, 3.5\%), kidney tumors (15 cases, $2.9 \%$ ), and upper gastrointestinal carcinomas (14 cases, 2.7\%) (Table 1). The tumor was multifocal in 97 cases (41.2\%), including 58 colorectal cancers, 19 neuroendocrine tumors, and 5 gastrointestinal stromal tumors.

The 509 cases comprised 274 biopsies and 235 resections. Liver resection of metastatic tumors was most commonly performed for colonic adenocarcinoma (142 cases, 60.4\%), followed by neuroendocrine tumors ( 29 cases, $12.3 \%$ ), gastrointestinal stromal tumors (11 cases, $4.6 \%$ ), female genital tract tumors (10 cases, $4.2 \%$ ), and kidney tumors ( 7 cases, 3\%). Surgical margins were negative in 172 cases of 235 resections $(73 \%)$ and positive in 63 cases. The most common procedure performed was partial hepatectomy including segmentectomy and lobectomy (184 cases), followed by excisional metastasectomy and wedge resections (46 cases). Five patients underwent total hepatectomy and transplantation for multifocal metastatic neuroendocrine tumor from the pancreas ( 3 cases), multifocal metastatic neuroendocrine tumor from the ileum (1 case), and multifocal metastatic gastrointestinal stromal tumor from the stomach (1 case), respectively.

Carcinoma was the most frequent tumor type (92\%). Adenocarcinoma, mostly from the gastrointestinal tract, was the most frequent type of carcinoma (309 cases, 60\%), followed by poorly differentiated carcinoma, carcinoma not otherwise specified, and neuroendocrine neoplasms. The remaining $8 \%$ of metastatic tumors were comprised of gastrointestinal stromal tumors (13 cases), sarcomas (6 cases), melanomas ( 7 cases), and germ cell tumors ( 7 cases).

Table I. Commonest Metastatic Liver Tumors.

\begin{tabular}{|c|c|c|c|c|c|}
\hline Primary tumor & & n (\% of total) & Males $(n)$, females $(n)$ & Age (years) & $\begin{array}{l}\text { Biopsies }(n), \\
\text { resections }(n)\end{array}$ \\
\hline Colon carcinoma & & 192 (38\%) & 124,68 & $28-89$ & 50,142 \\
\hline \multirow[t]{10}{*}{ Neuroendocrine tumors } & & $54(10.6 \%)$ & 29,25 & $30-86$ & 24,30 \\
\hline & Pancreas & 22 & 10,12 & $31-85$ & 6,16 \\
\hline & Stomach & 1 & $\mathrm{I}, 0$ & 54 & $\mathrm{I}, 0$ \\
\hline & Small intestine & 12 & 6,6 & $30-70$ & I, II \\
\hline & Colorectal & 3 & 2,1 & $4|-5|$ & 2,1 \\
\hline & Lung & 9 & 5,4 & $43-76$ & 9,0 \\
\hline & Bladder & 1 & $\mathrm{I}, 0$ & 86 & $\mathrm{I}, 0$ \\
\hline & Prostate & 2 & 2,0 & $64-68$ & 2,0 \\
\hline & Testis $^{\mathrm{a}}$ & I & $\mathrm{I}, 0$ & 50 & 0,1 \\
\hline & Unknown & 3 & $\mathrm{I}, 2$ & $60-7 \mid$ & 2,1 \\
\hline Breast carcinoma & & $50(9.8 \%)$ & I, 49 & $32-84$ & 48,2 \\
\hline Pancreatobiliary carcinoma & & $48(9.4 \%)$ & 23,25 & $38-84$ & 47,1 \\
\hline Tumors of female genital tract & & $27(5.3 \%)$ & 0,27 & $22-79$ & 17,10 \\
\hline Lung carcinoma & & $18(3.5 \%)$ & 7,11 & $33-84$ & 17,1 \\
\hline Kidney tumors & & $15(2.9 \%)$ & 5,10 & $18-84$ & 8,7 \\
\hline Upper gastrointestinal tract carcinoma & & $14(2.7 \%)$ & 10,4 & $59-82$ & 12,2 \\
\hline
\end{tabular}

${ }^{a}$ Neuroendocrine tumor arising in metastatic germ cell tumor. 


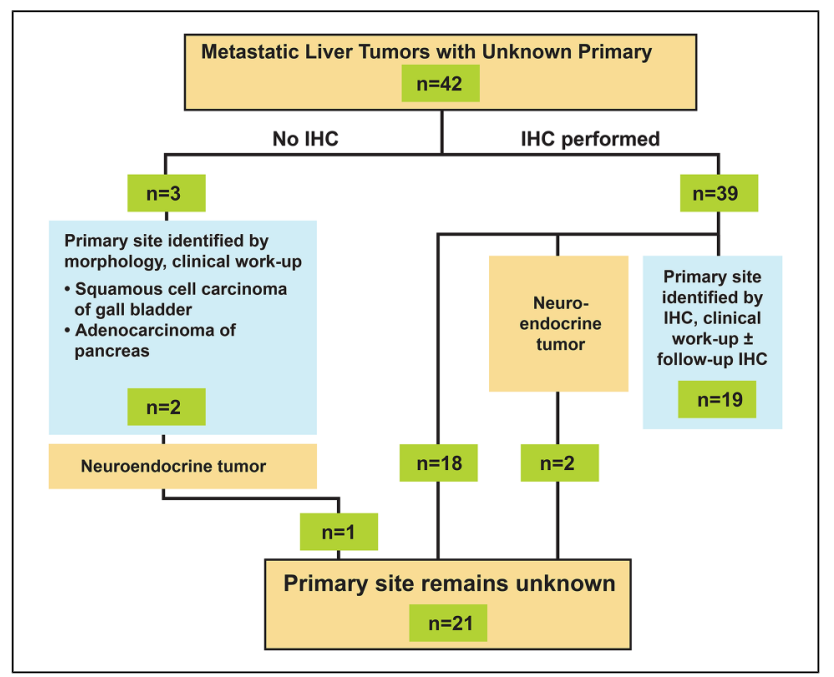

Figure I. Immunohistochemical and clinical workup of liver metastases with unknown primary allowed identification of the primary site in $50 \%$ of cases.

The site of the primary tumor was known at the time of biopsy or resection in all but 42 cases (Figure 1, Table 2). In 180 cases (35\%), the primary tumor had been previously biopsied and in 213 cases $(41.8 \%)$, the site of the primary tumor was known by imaging or other clinical work-up. The primary tumor was concomitantly resected in 74 cases (14.5\%). Of the 42 cases of unknown primary, 3 cases were diagnosed morphologically on biopsy as squamous cell carcinoma, possible pancreatobiliary adenocarcinoma, and well-differentiated neuroendocrine tumor, respectively. Further clinical work-up showed primary squamous cell carcinoma of the gallbladder tumor in the first case and a pancreatic adenocarcinoma in the second case. In the third case, the primary site of the neuroendocrine tumor was not found.

IHC was performed in the remaining 39 cases. The primary site was determined by IHC in 7 cases and in an additional 2 cases, a diagnosis of the well-differentiated neuroendocrine tumor was rendered. The primary site in the latter 2 cases could not be further determined by a detailed clinical work-up. In 12 cases, IHC suggested possible primary sites, which were then elucidated by follow-up clinical investigations; a second round of confirmatory IHC was performed in 3 of these 12 cases. In 18 cases, the primary site remained unknown; 9 of these patients died before the primary site could be identified and the remaining 9 were lost to follow-up. Molecular testing, performed in 1 case to identify the primary site, suggested a primary renal tumor. However, no renal mass was found. In 2 other patients with the unknown primary site, molecular testing was performed to identify targetable mutations. One case showed no targetable mutations whereas the second tumor contained several potential molecular targets.
IHC was performed in 23\% (120 of 509 cases) of metastatic liver lesions (Table 3 ). In cases where the primary was already known, IHC was used to confirm the diagnosis either because the morphological features were equivocal or because the clinical information was not robust. Additionally, IHC was performed for histological classification of lung tumors, and for biomarker expression analysis of breast carcinomas.

Immunohistochemical stains used most frequently were those for keratins (AE1/AE3, cytokeratin 7 [CK7], CK20, and p63), transcription factors (CDX2, TTF1, GATA3, and PAX8), neuroendocrine markers (synaptophysin and chromogranin), and prostatic markers (PSA, PSAP, $\alpha$-methyacyl CoA racemase AMACR, and p501s) and hormone receptors (estrogen receptor and progesterone receptor) (Table 2 and Supplemental Tables 4, 5).

\section{Discussion}

There are several population studies based on SEER data that detail the incidence and clinical characteristics of liver metastasis from specific organs or organ systems. ${ }^{1-5}$ However, data on the overall distribution and clinicopathologic characteristics of metastatic liver tumors are hard to come by. Pickren et $\mathrm{al}^{7}$ published their results from a series of 9700 autopsies performed between 1959 and 1979, whereas diSibio and French ${ }^{6}$ provided data on metastatic patterns of cancers from autopsies performed between 1914 and 1943. A more recent study published the incidence and age/gender distribution of metastatic liver tumors over a 10-year period based on data from a nationwide registry of histopathology and cytopathology in the Netherlands. ${ }^{8}$ In this European study, resections constituted one-fifth and biopsies constituted the remaining $80 \%$ of cases. This contrasts sharply with our cohort in which the number of resections almost matched (46\%) the number of biopsies for metastatic liver tumors. This finding was counterintuitive as tissue biopsies represent essential diagnostic tools and the liver is an easily accessible site for percutaneous tissue procurement. Furthermore, as liver metastases constitute stage IV cancer, surgical resection would seem to be futile in late-stage disease. In actuality, however, our study reflects contemporaneous organ-specific paradigms for diagnosis and management of metastatic liver disease in oncologic practice.

Population- and autopsy-based data reflect the natural history of tumors and their propensity for organo-tropic metastasis. Data from the nationwide pathology registry in the Netherlands reflects the role of biopsies in diagnosis of metastatic liver diseases. In contrast, our cohort reflects the association of our surgical pathology practice with an NCI-designated cancer institute, with additional specialization and expertise in liver surgery. Thus, we receive a large number of resections for metastatic liver tumors because metastasectomy from a variety of primary tumors is 
Table 2. Metastatic Liver Tumors With Initially Unknown Primary Site $(n=42)$.

\begin{tabular}{|c|c|c|c|}
\hline Primary tumor & Case & Positive markers & Negative markers \\
\hline \multicolumn{4}{|c|}{ Diagnosis rendered by immunohistochemistry $(n=7)$} \\
\hline \multirow[t]{2}{*}{ Breast } & $\mathrm{I}$ & CK7, GATA3 & $\begin{array}{l}\text { CK20, TTFI, CDX2, PAX8, ER, PR, glypican3, } \\
\text { synaptophysin, chromogranin }\end{array}$ \\
\hline & 2 & mammoglobin, ER, PR, GATA3 & - \\
\hline \multirow[t]{4}{*}{ Colon } & I & CK20, CDX2 & - \\
\hline & 2 & CK20, CDX2 & - \\
\hline & 3 & $\mathrm{CK} 20, \mathrm{CD} \times 2$ & CK7 \\
\hline & 4 & CK20, CDX2 & CK7, TTFI \\
\hline Lung adenocarcinoma & $\mathrm{I}$ & CK7, TTFI, napsin & - \\
\hline \multicolumn{4}{|c|}{ Diagnosis rendered by further clinical evaluation $(n=12)$ (follow-up immunohistochemistry in bold italics) } \\
\hline Colon & $\mathrm{I}$ & CDX2, CEA & CK7, CK20, PSA, P50Is \\
\hline Lung (squamous) & I & CK5/6, P63 & TTFI, napsin \\
\hline \multirow[t]{4}{*}{ Upper Gl } & $\mathrm{I}$ & AEI/AE3 & TTFI, CDX2, glypican3, arginase \\
\hline & 2 & CK7, CDX2 & $\begin{array}{l}\text { CK20, TTFI, PAX8, ER, PR, synaptophysin, } \\
\text { chromogranin }\end{array}$ \\
\hline & 3 & CDX2, MOC3I, glypican3 (focal) & CK7, CK20, TTFI, arginase, HSA, PSA, PSAP, P50Is \\
\hline & 4 & AEI/AE3, SALL4 & CK7, CK20, CDX2, TTFI, PSA, SI00, GATA3 \\
\hline Prostate & I & CDX2, PSAP, P50Is, AMACR & CK7, CK20, TTFI, PSA \\
\hline Thyroid (medullary) & 1 & $\begin{array}{l}\text { CK7, CEA, TTFI, synaptophysin, } \\
\text { chromogranin, calcitonin }\end{array}$ & Thyroglobulin, CK5/6, CDX2, CK20 \\
\hline Mesothelioma & I & $\begin{array}{l}\text { AEI/AE3, vimentin, CA9, RCC (focal), } \\
\text { GATA3, TFE3, WTI, calretinin }\end{array}$ & $\begin{array}{l}\text { PAX8, arginase, HSA, inhibin, SI00, DOGI, } \\
\text { CDII7, CD3I, TTFI, P63, SMA,HMB45 }\end{array}$ \\
\hline Kidney & I & $\begin{array}{l}\text { AEI/AE3, MOC3I, vimentin, RCC (focal), } \\
\text { PAX2 (focal), PAX8 }\end{array}$ & $\begin{array}{l}\text { CK7, CK20, P63, CK5/6, napsin, CEA, WTI, } \\
\text { CDX2, mammoglobin, ER, PR, inhibin, } \\
\text { calretinin, melanA }\end{array}$ \\
\hline Ovary & I & AEI/AE3 & Glypican3, arginase \\
\hline Pancreas NEC & I & Synaptophysin, CDX2 (focal) & Chromogranin, CK7, CK20 \\
\hline \multicolumn{4}{|c|}{ Diagnosis suggested on H\&E, confirmed by clinical work-up $(n=2)$} \\
\hline $\begin{array}{l}\text { Pancreatobiliary } \\
\text { adenocarcinoma }\end{array}$ & I & Immunohistochemisty not performed & \\
\hline $\begin{array}{l}\text { Gallbladder } \\
\text { adenosquamous tumor }\end{array}$ & I & Immunohistochemistry not performed & \\
\hline \multicolumn{4}{|c|}{ Diagnosis of NET/NEC rendered by immunohistochemistry but primary site unknown $(n=3)$} \\
\hline NET & $\mathrm{I}$ & Synaptophysin, Ki67: I\% & CK7, CK20, ER, PR, GATA3 \\
\hline NET & 2 & Ki67: $1 \%$ & \\
\hline NEC & 3 & Synaptophysin, chromogranin, TTF-I (weak) & ER, PR, GATA3, HER2, HSA, arginase \\
\hline \multicolumn{4}{|c|}{ Primary tumor unknown; patients lost to follow up $(n=9)$ or died $(n=9)$} \\
\hline \multirow[t]{11}{*}{ Adenocarcinoma } & $\mathrm{I}$ & CK7 & CK20, TTFI, CDX2, Napsin, WTI, ER, PR \\
\hline & 2 & $\begin{array}{l}\text { CK7 (focal), CK20 (focal), CDX2, synaptophysin } \\
\text { (weak focal), chromogranin (focal) }\end{array}$ & \\
\hline & 3 & MOC-3I, BerEP4, CDX2, synaptophysin (focal) & AEI/AE3, CK7, CK20, chromogranin \\
\hline & 4 & CK7, CDX2 & CK20, TTFI, napsin, \\
\hline & 5 & AEI/AE3, CK7 & $\begin{array}{l}\text { CK20, CDX2, GATA3, ER, PR, synaptophysin, } \\
\text { chromogranin }\end{array}$ \\
\hline & 6 & CDX2, GATA3 (focal) & CK7, CK20, TTFI \\
\hline & 7 & $\mathrm{CD} \times 2$ & CK7, CK20, TTFI, PSA, PSAP, p50Is, \\
\hline & 8 & CK7 & CK20, CDX2, TTFI, Napsin, P63 \\
\hline & 9 & AEI/AE3 & CK7, CK20, CDX2, TTFI \\
\hline & 10 & AEI/AE3, CAM5.2, CK7 & $\begin{array}{l}\text { CK20, CDX2, TTFI, p63, glypican3, arginase, } \\
\text { inhibin }\end{array}$ \\
\hline & $\begin{array}{l}11 \\
12\end{array}$ & $\begin{array}{l}\text { MOC3I, CK7, CKI9, HSA } \\
\text { SMAD4 }\end{array}$ & $\begin{array}{l}\text { CK20, TTFI, CDX2, PSA, gypican3, arginase } \\
\text { TTFI, napsin, CDX2, PAX8, arginase, } \\
\text { synaptophysin, chromogranin }\end{array}$ \\
\hline
\end{tabular}


Table 2. (continued)

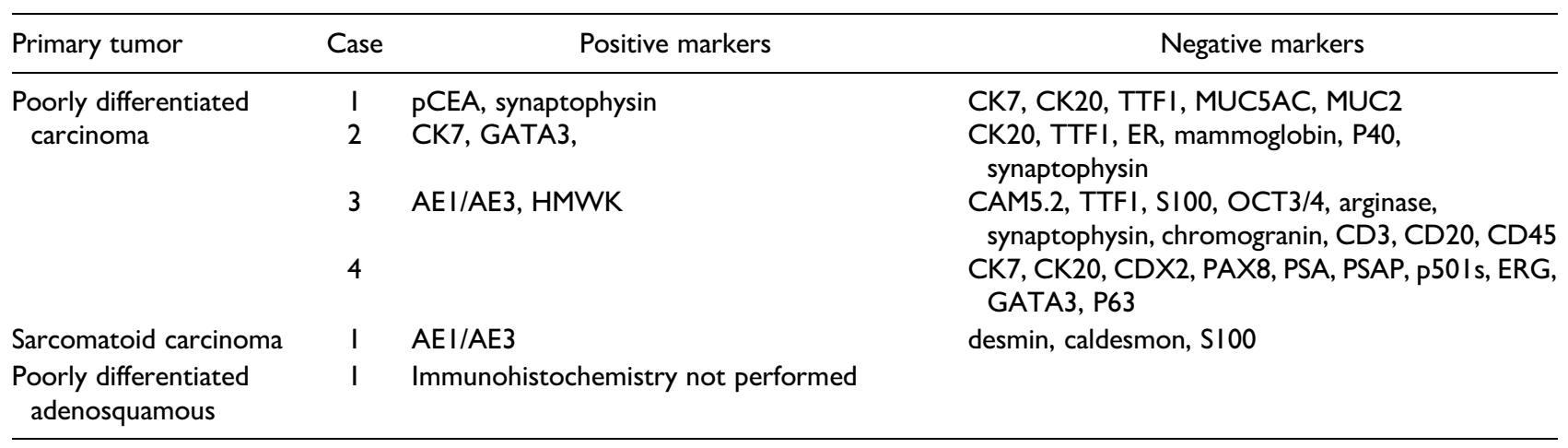

Abbreviations: CK, cytokeratin; Gl, gastrointestinal tract, H\&E, hematoxylin and eosin; NET/NEC, neuroendocrine tumor/neuroendocrine carcinoma.

Table 3. Use of Immunohistochemistry for Metastatic Liver Tumors.

\begin{tabular}{lcc}
\hline Primary site & $\begin{array}{c}\text { Immunohistochemistry } \\
\text { performed }(\mathrm{n})\end{array}$ & $\begin{array}{c}\text { Total } \\
\text { cases }(\mathrm{n})\end{array}$ \\
\hline Unknown primary & 39 & 42 \\
Breast & 31 & 50 \\
Lung & 13 & 27 \\
Colorectal & 13 & 192 \\
Female genital tract & 9 & 27 \\
Kidney & 6 & 15 \\
Upper gastrointestinal tract & 6 & 15 \\
Prostate & 5 & 9 \\
Miscellaneous & 120 & 509 \\
\hline
\end{tabular}

associated with improved overall, and in many instances, disease-free, long-term survival. Advances in anesthetic technique and critical care have reduced mortality of liver resections in tertiary care centers to $<5 \%$. Furthermore, advances in preoperative and intraoperative imaging allow a more accurate assessment of the extent of liver involvement and therefore, the extent of resectability of liver metastases. In addition, constantly evolving chemotherapy regimens have made it possible to downstage tumors and render more of them resectable. ${ }^{9}$ Finally, for most tumors, liver metastasectomy performed simultaneously with resection of the primary tumor, as seen in 74 of our cases, has comparable morbidity, mortality, and long-term outcomes, as staged procedures.

The largest body of literature and cumulative experience exists for liver resection of metastatic colorectal carcinomas, metastatic neuroendocrine tumors, and metastatic gastrointestinal stromal tumors. To wit, resections for these 3 metastatic lesions constituted more than three-fourths $(78 \%)$ of liver resections in our study. An analysis of over 10000 patients with colorectal cancer in the SEER database showed that $80 \%$ of patients had only liver metastases and $12.8 \%$ had both liver and lung metastases. In this cohort, metastasectomy improved the 3 years overall survival in those who had liver metastases but not those with lung or combined lung or liver metastases. ${ }^{1}$ Liver metastasis is the most important predictor of survival in patients with neuroendocrine tumors. Hormone secretion and hepatic decompensation due to parenchymal replacement are the main causes of morbidity and mortality in these patients. Complete surgical hepatic resection offers better survival compared with transarterial embolization or medical treatment, allowing median survival of 125 months with overall survival of $74 \%$ and $51 \%$ at 5 and 10 years, respectively. Even incomplete resection with the aim of debulking is beneficial to many patients. ${ }^{10,11}$ Similarly, liver metastasis affect survival in patients with gastrointestinal stromal tumor; the 5 years overall survival for those with and without liver metastasis was reported to be $39.9 \%$ and $75.5 \%$, respectively. ${ }^{12}$ The vast majority of patients with gastrointestinal stromal tumors have only liver metastasis. Because long term complete remissions are rare with imatinib alone, the current standard of care combines imatinib with complete surgical resection. ${ }^{13}$

The influence of standard of care and institutional specialization is further exemplified by the number of metastatic neuroendocrine tumors in our cohort, which constitute the second most common tumor in our study. These tumors were not as common in the study based on the national pathology registry of the Netherlands, in which metastatic breast and pancreatic tumors were the second and third commonest tumors after metastatic colorectal carcinoma. ${ }^{8}$ In our study, metastatic breast and pancreatic carcinomas followed neuroendocrine tumors as the next common tumor types. In contrast to metastatic colorectal carcinomas, metastatic neuroendocrine tumors, and metastatic gastrointestinal tumors however, metastatic breast and pancreatic tumors were encountered as biopsies rather than as resection material. This is because chemotherapy and local ablative therapies take precedence over resection for metastatic breast cancer. ${ }^{14}$ Patients 
with pancreatic carcinoma who have isolated liver metastasis have a worse prognosis than those who have isolated lung or distant nodal metastasis, ${ }^{15}$ and the role of metastasectomy is only beginning to be defined, albeit only for a very select group of patients. ${ }^{16-18}$ Similarly, the prognosis is uniformly poor for liver metastases from tumors of the lung or upper gastrointestinal tract that were rarely seen as resection specimens. ${ }^{4,19,20}$

Led by refinements in surgical, perioperative, and imaging modalities, resection is slowly being extended to other tumor types in carefully selected patients, and surgical pathologists can expect to see an increasing number of resections for metastatic liver tumors in the years to come. Existing data suggests that prognosis is best for genitourinary tumors, particularly renal cell carcinoma, for which survival outcomes match those of colorectal carcinoma metastatic to the liver. ${ }^{20-24}$

Immunohistochemical stains were performed in less than a quarter of the total number of cases (23\%). Utilization of IHC in metastatic liver tumors is influenced by the type of practice (academic- vs community-based practices, generalist vs specialist reporting), prior information of the primary tumor site, and the clinical need to assess tissue biomarker status for therapeutic management. ${ }^{25}$ In our study, the primary tumor site was known in the majority of cases through clinical history provided on the requisition form, or by accessing the electronic medical record. A study on the utilization of IHC in metastatic liver tumors found that pathologists are 1.5 times more likely to perform IHC if the primary tumor site is not known. ${ }^{25}$ For example, in our practice, pancreatic tumors are diagnosed by fine-needle aspiration cytology obtained by endoscopic ultrasound-guided biopsies of the pancreas itself. This approach spares us the extreme difficulty of differentiating metastatic pancreatic adenocarcinoma from other metastatic liver tumors and, especially, from primary intrahepatic cholangiocarcinoma. Therefore, in this cohort, IHC was performed on only 1 case of metastatic pancreatic carcinoma in the liver.

Of the cases with known primary tumor site, IHC was performed in our practice mostly in metastatic breast, colon, and lung carcinomas. In these cases, biomarker analyses provided additional information relevant to clinical management. In both breast and lung carcinomas, the expression of prognostic markers in metastatic lesions is known to differ from the primary tumor in numerous cases. ${ }^{12,26}$ In cases where IHC would not have provided additional clinically relevant information, dual commitments to cost containment and quick turn-around time might have influenced the pathologists' decision to forgo IHC testing. ${ }^{25}$

IHC was performed in 39 of the 42 cases in which the primary tumor site was not known; it helped to identify the primary tumor site in 19 cases and rendered a diagnosis of neuroendocrine tumor in an additional 3 cases. The primary tumor site remained unknown in $7.5 \%$ of cases in which IHC was used. This is slightly less than the $11 \%$ and $18 \%$ of cases, respectively, reported in 2 other studies on metastatic liver tumors in which IHC was used to identify the primary tumor site. The antibodies used in our practice for identifying the primary site are similar to those reported in other series. ${ }^{25,27}$

\section{Conclusions}

In summary, metastatic liver tumors in a surgical pathology practice are encountered as biopsies performed for diagnosis, or as resections performed for tumor debulking or with curative intent. Our practise is affiliated with an NCI-designated cancer center, and receives a large number of resections reflecting current standards of care and best practices in the management of metastatic liver tumors. In carefully selected patients, resection of liver metastases from a variety of primary tumors offers improved overall, and in many instances, improved disease-free survival. This is particularly true of the big 3: metastatic colorectal carcinomas, metastatic neuroendocrine tumors, and metastatic gastrointestinal stromal tumors, which constituted two-thirds of all our resection specimens. IHC of metastatic liver tumors serves 2 main functions: the first is to assist in finding the primary tumor site, and the second is to assess tissue biomarker expression and guide therapeutic management.

\section{Declaration of Conflicting Interests}

The authors declared no potential conflicts of interest with respect to the research, authorship, and/or publication of this article.

\section{Funding}

The authors received no financial support for the research, authorship, and/or publication of this article.

\section{Ethical Approval}

Approved by the Institutional Review Board of Indiana University School of Medicine.

\section{Informed Consent}

Waiver of informed consent was approved by the Institutional Review Board of Indiana University.

\section{Trial Registration}

Not applicable, because this article does not contain any clinical trials.

\section{ORCID iD}

Ayşe Armutlu iD https://orcid.org/0000-0001-9804-0454

\section{Supplemental Material}

Supplemental material for this article is available online. 


\section{References}

1. Siebenhuner AR, Guller U, Warschkow R. Population-based SEER analysis of survival in colorectal cancer patients with or without resection of lung and liver metastases. $B M C$ Cancer. 2020;20(1):246. doi:10.1186/s12885-020-6710-1

2. Wang S, Feng Y, Swinnen J, Oyen R, Li Y, Ni Y. Incidence and prognosis of liver metastasis at diagnosis: a pan-cancer population-based study. Am J Cancer Res. 2020;10(5): 1477-1517.

3. Yang DY, Wang X, Yuan WJ, Chen ZH. Metastatic pattern and prognosis of gastrointestinal stromal tumor (GIST): a SEER-based analysis. Clin Transl Oncol. 2019;21(12): 1654-1662. doi:10.1007/s12094-019-02094-y

4. Yang J, Zhang Y, Sun X, et al. The prognostic value of multiorgan metastases in patients with non-small cell lung cancer and its variants: a SEER-based study. J Cancer Res Clin Oncol. 2018;144(9):1835-1842. doi:10.1007/s00432-018-2702-9

5. Yao JC, Hassan M, Phan A, et al. One hundred years after "carcinoid": epidemiology of and prognostic factors for neuroendocrine tumors in 35,825 cases in the United States. $J$ Clin Oncol. 2008;26(18):3063-3072. doi:10.1200/JCO. 2007.15.4377

6. Disibio G, French SW. Metastatic patterns of cancers: results from a large autopsy study. Arch Pathol Lab Med. 2008; 132(6):931-939. doi:10.1043/1543-2165(2008)132[931: MPOCRF]2.0.CO;2

7. Pickren JW, Tsukada Y, Lane WW. Liver metastases: Analysis of autopsy data. Liver Metastases. Hall Medical Publishers; 1982.

8. de Ridder J, de Wilt JH, Simmer F, Overbeek L, Lemmens V, Nagtegaal I. Incidence and origin of histologically confirmed liver metastases: an explorative case-study of 23,154 patients. Oncotarget. 2016;7(34):55368-55376. doi:10.18632/oncotarget.10552

9. Bartlett EK, Simmons KD, Wachtel H, et al. The rise in metastasectomy across cancer types over the past decade. Cancer. 2015;121(5):747-757. doi:10.1002/cncr.29134

10. Frilling A, Clift AK. Therapeutic strategies for neuroendocrine liver metastases. Cancer. 2015;121(8):1172-1186. doi:10.1002/cncr.28760

11. Mayo SC, de Jong MC, Pulitano C, et al. Surgical management of hepatic neuroendocrine tumor metastasis: results from an international multi-institutional analysis. Ann Surg Oncol. 2010;17(12):3129-3136. doi:10.1245/s10434-010-1154-5

12. Deng LL, Deng HB, Lu CL, Gao G, Wang F, Yang Y. Differential molecular markers of primary lung tumors and metastatic sites indicate different possible treatment selections in patients with metastatic lung adenocarcinoma. Clin Transl Oncol. 2019;21(2):197-205. doi:10.1007/s12094-018-1906-4

13. Machairas N, Prodromidou A, Molmenti E, Kostakis ID, Sotiropoulos GC. Management of liver metastases from gastrointestinal stromal tumors: where do we stand? J Gastrointest Oncol. 2017;8(6):1100-1108. doi:10.21037/jgo.2017.08.08

14. Bale R, Putzer D, Schullian P. Local treatment of breast cancer liver metastasis. Cancers (Basel). 2019;11(9):1341. doi:10.3390/cancers 11091341
15. Oweira H, Petrausch U, Helbling D, et al. Prognostic value of site-specific metastases in pancreatic adenocarcinoma: a surveillance epidemiology and End results database analysis. World J Gastroenterol. 2017;23(10):1872-1880. doi:10. 3748/wjg.v23.i10.1872

16. Ghidini M, Petrillo A, Salati M, et al. Surgery or locoregional approaches for hepatic oligometastatic pancreatic cancer: myth, hope, or reality? Cancers (Basel). 2019;11(8):1095. doi:10.3390/cancers11081095

17. Lee RC, Kanhere H, Trochsler M, Broadbridge V, Maddern G, Price TJ. Pancreatic, periampullary and biliary cancer with liver metastases: should we consider resection in selected cases? World J Gastrointest Oncol. 2018;10(8):211220. doi:10.4251/wjgo.v10.i8.211

18. Voss N, Izbicki JR, Nentwich MF. Oligometastases in pancreatic cancer (synchronous resections of hepatic oligometastatic pancreatic cancer: disputing a principle in a time of safe pancreatic operations in a retrospective multicenter analysis). Ann Gastroenterol Surg. 2019;3(4):373-377. doi:10.1002/ ags3.12255

19. Riihimaki M, Hemminki A, Fallah M, et al. Metastatic sites and survival in lung cancer. Lung Cancer. 2014;86(1):78-84. doi:10.1016/j.lungcan.2014.07.020

20. Schiergens TS, Lüning J, Renz BW, et al. Liver resection for non-colorectal non-neuroendocrine metastases: where do we stand today compared to colorectal cancer? J Gastrointest Surg. 2016;20(6):1163-1172. doi:10.1007/s11605-016-3115-1

21. Holzner PA, Makowiec F, Klock A, et al. Outcome after hepatic resection for isolated non-colorectal, nonneuroendocrine liver metastases in 100 patients - the role of the embryologic origin of the primary tumor. $B M C$ Surg. 2018;18(1):89. doi:10.1186/s12893-018-0424-1

22. Uggeri F, Ronchi PA, Goffredo P, et al. Metastatic liver disease from non-colorectal, non-neuroendocrine, nonsarcoma cancers: a systematic review. World J Surg Oncol. 2015;13:191. doi:10.1186/s12957-015-0606-6

23. Pinotti E, Montuori M, Giani A, et al. Surgical treatment of liver metastases from kidney cancer: a systematic review. ANZ J Surg. 2019;89(1-2):32-37. doi:10.1111/ ans. 15000

24. Golse N, Adam R. Liver metastases from breast cancer: what role for surgery? Indications and results. Clin Breast Cancer. 2017;17(4):256-265. doi:10.1016/j.clbc.2016.12.012

25. Amin K, El-Rayes D, Snover D, Mettler T, Vogel RI, Khalifa MA. Patterns of immunohistochemistry utilization in metastases to the liver. Appl Immunohistochem Mol Morphol. 2019;27(6):441-447. doi:10.1097/pai.000 0000000000643

26. Curtit E, Nerich V, Mansi L, et al. Discordances in estrogen receptor status, progesterone receptor status, and HER2 status between primary breast cancer and metastasis. Oncologist. 2013;18(6):667-674. doi:10.1634/theoncologist. 2012-0350

27. Park JH, Kim JH. Pathologic differential diagnosis of metastatic carcinoma in the liver. Clin Mol Hepatol. 2019;25(1): 12-20. doi:10.3350/cmh.2018.0067 